Med. thorac. 1965;22:346

\title{
Discussion
}

\section{Paper of HILDING}

H. Boren (Saranac Lake): The action of the cilia of frogs is inhibited by small particles of carbon. When these particles themselves are too large to be moved by the cilia, viscid mucus will form and sweep the area in which the particles are lying.

A. Toigo (Hines): Healthy young men were given an aerosol of carbon particles labelled with radioactive gold. The 70 micron particles were too large to penetrate into the alveoli. Their clearance was followed by counters over the chest; it was found that normal lungs were $80 \%$ cleared in two hours. Emphysema and/or chronic bronchitis markedly deranged the clearance. One patient with a virus respiratory infection failed to clear all particles until four months had elapsed. Smokers and non-smokers without disease have the same clearance time in those cases studied so far. During a test each subject receives about 20 microcuries, about twice the dose delivered by a chest $\mathrm{x}$-ray. This amount of radiation is not felt to be sufficient to impair clearance.

C. Stuart-Harris (Sheffield): Ciliated epithelium is often selectively damaged during viral infections. 